\title{
Probing Triplet State Properties of Organic Chromophores via Design and Synthesis of Os(II)-Diketonate Complexes: The Triplet State Intramolecular Charge Transfer
}

\author{
Jen-Kan Yu, ${ }^{\dagger}$ Yi-Ming Cheng, ${ }^{\dagger}$ Ya-Hui Hu, ${ }^{\dagger}$ Pi-Tai Chou,,${ }^{*} \dagger$ Yao-Lun Chen, ${ }^{*}$ \\ Shin-Wun Lee, ${ }^{*}$ and Yun Chi*,* \\ Department of Chemistry, National Taiwan University, Taipei 106, Taiwan, and Department of Chemistry, \\ National Tsing Hua University, Hsinchu 30043, Taiwan
}

Received: October 14, 2004

\begin{abstract}
We report the probe of specific triplet state properties of organic chromophores that are otherwise inaccessible in low viscous solution. The prototypical example demonstrated here is $\left[\mathrm{Os}(\mathrm{CO})_{3}(\mathrm{Cl})(\mathrm{NDP})\right](\mathbf{1})((\mathrm{NDP}) \mathrm{H}=$ 2-naphthyl-7-dimethylanilino-1,3-propanedione), which, upon electronic excitation, undergoes intramolecular charge transfer in both $S_{1}$ and $T_{1}$ manifolds of NDP. The dipolar changes in $S_{1}$ and $T_{1}$ monitored via the solvatochromism for both fluorescence and phosphorescence were deduced to be 18.0 and $11.9 \mathrm{D}$, respectively. The appreciable difference in the dipolar change can be qualitatively rationalized by different extents of charge-transfer character between $S_{1}$ and $T_{1}$ states. The results led to the probe of other reactions in triplet manifold feasible.
\end{abstract}

Third-row heavy metal containing luminescent complexes have increasingly gained attention due to their potential applications in electroluminescent devices. ${ }^{1}$ The strong spin-orbit coupling effectively promotes an intersystem crossing from singlet excited states to lower triplet emitting states, namely ${ }^{3} \mathrm{MLCT},{ }^{3} \pi \pi^{*}$, or a mixture of the two. ${ }^{2}$ The resulting materials are suitable for use in the preparation of OLEDs with unprecedented phosphorescence efficiencies attained by harnessing both triplet and singlet excitons, theoretically giving $100 \%$ internal quantum efficiency. ${ }^{3}$

We here would like to advance another angle of approach toward probing the fundamentals of the triplet-state properties based on the design of Os(II) complexes. Our strategy is to functionalize ligands connected to the heavy metal, so that the lowest triplet-state energy level can be fine tuned and thus lie mainly in the $\pi \pi^{*}$ (or $n \pi^{*}$ ) chromophore of the designated ligand. On this basis, one can ingeniously design a special type of triplet-state reaction, the associated properties of which can thus be dictated by the prominent phosphorescence. A case in point is the triplet state intramolecular charge transfer (TSICT) reaction, ${ }^{4}$ the investigation of which is rare due to the spin forbidden nature of the $\mathrm{T}_{1} \rightarrow \mathrm{S}_{0}$ transition. Several methods have been employed to circumvent the lack of phosphorescence in solution. The micelle or polymer encapsulated organic chromophore, together with the external heavy atom effect, may resolve room-temperature phosphorescence. ${ }^{5}$ However, the resulting heterogeneous and rigid environments make studies of the solvation-associated properties impractical. As a result, photophysical properties associated with TSICT, such as the solvation relaxation dynamics as well as the steady-state consequence, e.g., the phosphorescence solvatochromism, are not attainable in low viscous solvents. To achieve this goal, we have designed and synthesized an asymmetrical $\beta$-diketonate ligand, composed of an $N, N$-dimethylaniline moiety that serves as the electron donor, ${ }^{6}$ and obtained the corresponding Os(II) carbonyl-metal complexes. The addition of back $\pi$-bond

* Corresponding author. E-mail: chop@ntu.edu.tw; ychi@mx.nthu.edu.tw.

National Taiwan University.

$\doteqdot$ National Tsing Hua University. donating carbonyl ligands should further increase the $d-d$ energy gap, ${ }^{7}$ so that the organic chromophore of interest, i.e., $\beta$-diketonate, is expected to lie in the lower-lying excited states. The $\beta$-diketonate ligand with both donor and acceptor substituents was synthesized through a Claisen condensation reaction, in which treatment of 2-acetonaphthone and 4-dimethylaminobenzoic acid ethyl ester in the presence of a strong base $\mathrm{NaH}$ gives the desired diketone, 2-naphthyl-7-dimethylanilino-1,3propanedione (NDP)H. To prepare the Os(II)-metal complexes, we selected the literature method designed for the related dibenzoylmethanate complex $\left[\mathrm{Os}(\mathrm{CO})_{3}(\mathrm{tfa})(\mathrm{dbm})\right]$, tfa $=$ $\mathrm{CF}_{3} \mathrm{CO}_{2}{ }^{-}$and $(\mathrm{dbm}) \mathrm{H}=$ dibenzoylmethane. ${ }^{8}$ In this approach, heating the finely pulverized solid-state mixture of (NDP)H and osmium dimer $\left[\mathrm{Os}_{2}(\mathrm{CO})_{6}(\mathrm{tfa})_{2}\right]$ afforded $\left[\mathrm{Os}(\mathrm{CO})_{3}(\mathrm{tfa})(\mathrm{NDP})\right]$ (1a). Treatment of $\mathbf{1 a}$ with $\mathrm{NaCl}$ in refluxing $\mathrm{THF}$ solution afforded the ligand substitution product $\left[\mathrm{Os}(\mathrm{CO})_{3}(\mathrm{Cl})(\mathrm{NDP})\right]$ (1) in $\sim 80 \%$ yield. Moreover, treatment of (NDP)H with $\mathrm{BPh}_{3}$ in THF solution at room temperature gave the respective $\mathrm{BPh}_{2}$ complex 2 in $90 \%$ yield (see Supporting Information).

Figure 1 shows absorption and emission spectra of complex $\mathbf{1}$ in various solvents. As solvent polarity increased, in contrast to a slight bathochromic shift of the absorption spectral features, a drastic solvent-polarity dependence in emission spectra was observed. In aerated cyclohexane, 1 exhibited distinct dual emission with $\lambda_{\max }$ at 470 and $570 \mathrm{~nm}$. While the intensity of the $470 \mathrm{~nm}$ band remained unchanged, the $570 \mathrm{~nm}$ band revealed drastic $\mathrm{O}_{2}$-concentration dependence and became dominant in the degassed solution (see Figure 1). The $\mathrm{O}_{2}$ quenching rate was calculated to be as large as $1.4 \times 10^{9} \mathrm{M}^{-1} \mathrm{~s}^{-1}$ (Supporting Information). Accordingly, the 470 and $570 \mathrm{~nm}$ bands in 1 unambiguously are ascribed to the fluorescence and phosphorescence, respectively. Table 1 lists the photophysical properties of the studied complexes. In degassed cyclohexane the phosphorescence lifetime for 1 was measured to be $\sim 60 \mu \mathrm{s}$. This, in combination with a near unity of the phosphorescence quantum yield, renders a radiative lifetime of $\sim 60 \mu \mathrm{s}$, which is much shorter than that of the triplet $\pi \pi^{*}$ for the free NDPH ligand ( $\sim 0.1 \mathrm{~s}$ measured in the $77 \mathrm{~K}$ methylcyclohexane glass). The results support the enhancement of the $T_{1} \rightarrow S_{0}$ transition 


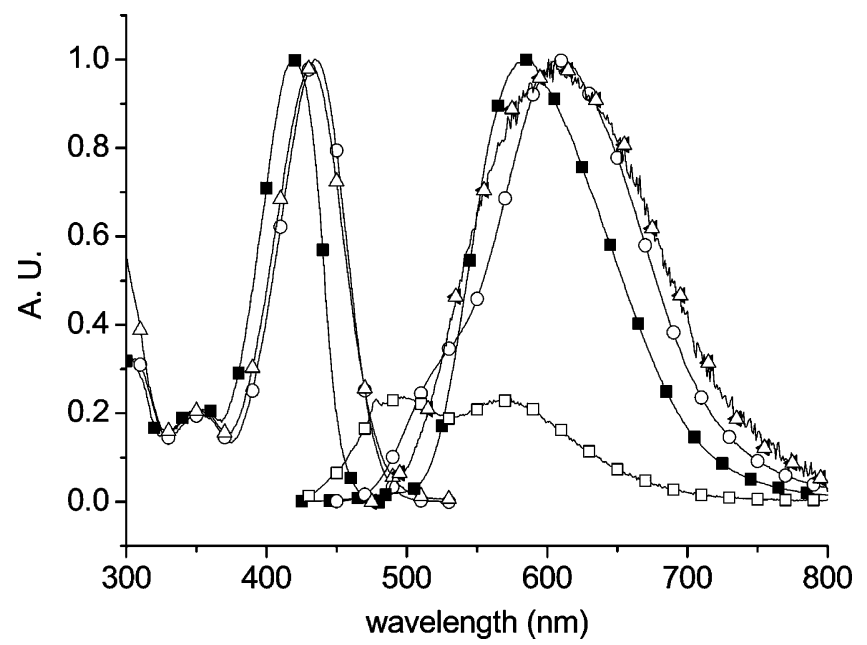

Figure 1. Absorption and steady-state emission spectra of $\mathbf{1}$ at $298 \mathrm{~K}$ degassed cyclohexane (-口-), dichloromethane (-O-), and acetonitrile $(-\triangle-) .(-\square-)$ denotes the emission spectrum of $\mathbf{1}$ in aerated cyclohexane.

TABLE 1: Photophysical Properties of Complexes 1, 1a, and 2

\begin{tabular}{|c|c|c|c|c|c|c|c|}
\hline & & $\lambda_{\mathrm{abs}}$ & $\begin{array}{c}\lambda_{\mathrm{em}}^{\max a} \\
(\mathrm{~nm})\end{array}$ & $\begin{array}{c}\lambda_{\mathrm{em}}^{\max b} \\
(\mathrm{~nm})\end{array}$ & $\Phi^{b, c}$ & $\tau_{\mathrm{f}}^{a}$ & $\tau_{\mathrm{p}}^{b}$ \\
\hline \multirow[t]{5}{*}{1} & $\mathrm{C}_{6} \mathrm{H}_{12}$ & 424 & 470,570 & 574 & $\sim 1$ & $15.4(\mathrm{ps})^{d}$ & $60(\mu \mathrm{s})$ \\
\hline & $\mathrm{C}_{6} \mathrm{H}_{6}$ & 432 & 508 & 593 & 0.84 & $15.6(\mathrm{ps})^{d}$ & $125(\mu \mathrm{s})$ \\
\hline & THF & 430 & 537 & 609 & 0.40 & 104 (ps) & $74(\mu \mathrm{s})$ \\
\hline & $\mathrm{CH}_{2} \mathrm{Cl}_{2}$ & 436 & 543 & 611 & 0.58 & $110(\mathrm{ps})$ & $86(\mu \mathrm{s})$ \\
\hline & $\mathrm{CH}_{3} \mathrm{CN}$ & 422 & 580 & 649 & 0.005 & $22.0(\mathrm{ps})^{d}$ & $70(\mu \mathrm{s})$ \\
\hline \multirow[t]{3}{*}{$1 \mathrm{a}$} & $\mathrm{C}_{6} \mathrm{H}_{12}$ & 419 & 497,583 & 577 & $\sim 1$ & $5.13(\mathrm{ps})^{d}$ & $105(\mu \mathrm{s})$ \\
\hline & $\mathrm{CH}_{2} \mathrm{Cl}_{2}$ & 435 & 532 & 603 & 0.61 & 113 (ps) & $201(\mu \mathrm{s})$ \\
\hline & $\mathrm{CH}_{3} \mathrm{CN}$ & 432 & 575 & 646 & 0.015 & $8.52(\mathrm{ps})^{d}$ & $50(\mu \mathrm{s})$ \\
\hline \multirow[t]{3}{*}{2} & $\mathrm{C}_{6} \mathrm{H}_{12}$ & 434 & 467 & 467 & 0.12 & 1.23 (ns) & \\
\hline & $\mathrm{CH}_{2} \mathrm{Cl}_{2}$ & 461 & 537 & 537 & 0.39 & 1.07 (ns) & \\
\hline & $\mathrm{CH}_{3} \mathrm{CN}$ & 466 & 572 & 572 & 0.005 & 0.21 (ns) & \\
\hline
\end{tabular}

${ }^{a}$ Data were obtained in aerated solution. ${ }^{b}$ Data were obtained in degassed solution (via three freeze-pump-thaw cycles). ${ }^{c}$ Quantum yield was obtained for fluorescence plus phosphorescence. ${ }^{d}$ Due to the system response of $30 \mathrm{ps}$, these values obtained from curve fitting are subject to large uncertainties.

dipole in $\mathbf{1}$ due to the Os heavy atom effect. Conversely, the fluorescence lifetime of $\mathbf{1}$ in cyclohexane is beyond the system response of $30 \mathrm{ps}$ (see Table 1). In comparison, the boron complex 2 (see Scheme 1) bearing the same NDP ligand exhibits a unique fluorescence, the lifetime of which is as long as 1.23 ns in cyclohexane. Assuming that the relaxation dynamics of $S_{1}$ in $\mathbf{1}$ is dominated by rate of $S_{1} \rightarrow T_{n}$, the results also manifest the enhancement of spin-orbit coupling on the $\mathrm{S}_{1} \rightarrow \mathrm{T}_{n}$ intersystem crossing due to the Os heavy atom effect.

In degassed, polar solvents, fluorescence and phosphorescence strongly overlap, giving rise to a broad emission (Figure 1). Fortunately, as shown in Table 1, the different relaxation dynamics of fluorescence and phosphorescence allow us to temporally resolve each component (Supporting Information). In the degassed solution, the spectrum taken at a delayed time of $5 \mu \mathrm{s}$ after the excitation ensures the acquisition of phosphorescence free from fluorescence interference. Conversely, gating the time-resolved spectrum at the first $\sim 10$ ns should obtain a nearly phosphorescence-free fluorescence. The results shown in Figure 2 clearly reveal remarkable solvent dependency, and the plot of peak frequency for both fluorescence and phosphorescence is linearly proportional to solvent polarity $(\Delta f)^{9}$ (see inset of Figure 2). Accordingly, changes in dipole moment with respect to the ground state were deduced to be 18.0 and $11.9 \mathrm{D}$ for $\mathrm{S}_{1}$ and $\mathrm{T}_{1}$, respectively, with the Lippert-Mataga equation. ${ }^{10, \mathrm{SI}}$

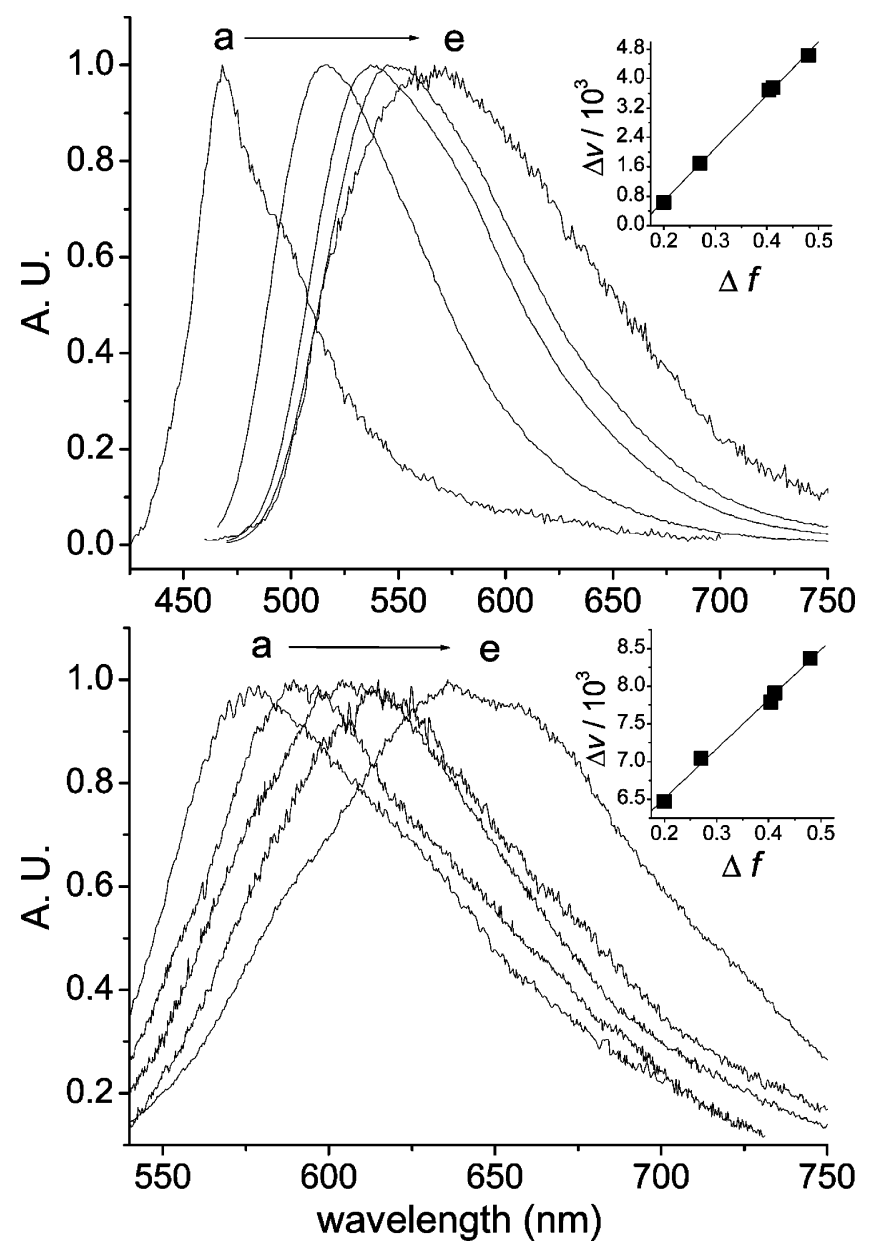

Figure 2. Time-gated fluorescence (upper) and phosphorescence (lower) of 1 in degassed (a) $\mathrm{C}_{6} \mathrm{H}_{12}$, (b) $\mathrm{C}_{6} \mathrm{H}_{6}$, (c) $\mathrm{CH}_{2} \mathrm{Cl}_{2}$, (d) THF, and (e) $\mathrm{CH}_{3} \mathrm{CN}$. (inset) The plot of peak frequency versus solvent polarity $\Delta f$.

SCHEME 1: Complexes 1-2 and the Proposed Prototypes to Study Charge (3) and Proton (4) Transfer in the Triplet State

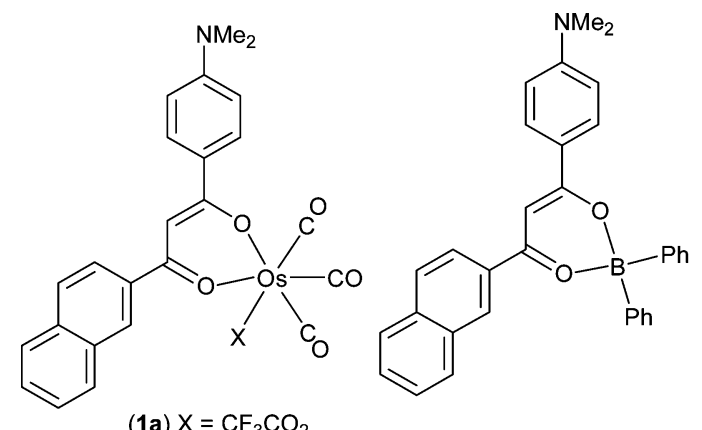

(1a) $\mathrm{X}=\mathrm{CF}_{3} \mathrm{CO}_{2}$

(1) $\mathrm{X}=\mathrm{Cl}$

(2) $\left[(\mathrm{NDP}) \mathrm{BPh}_{2}\right]$

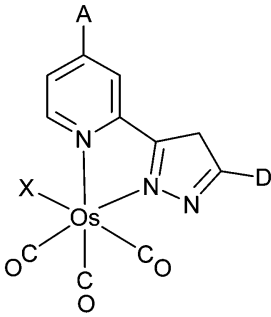<smiles>[X]C([X])(O)C([X])(C)O</smiles> 
TABLE 2: Calculated Electronic Transitions and Associated Frontier Orbitals for Complex $1^{a}$

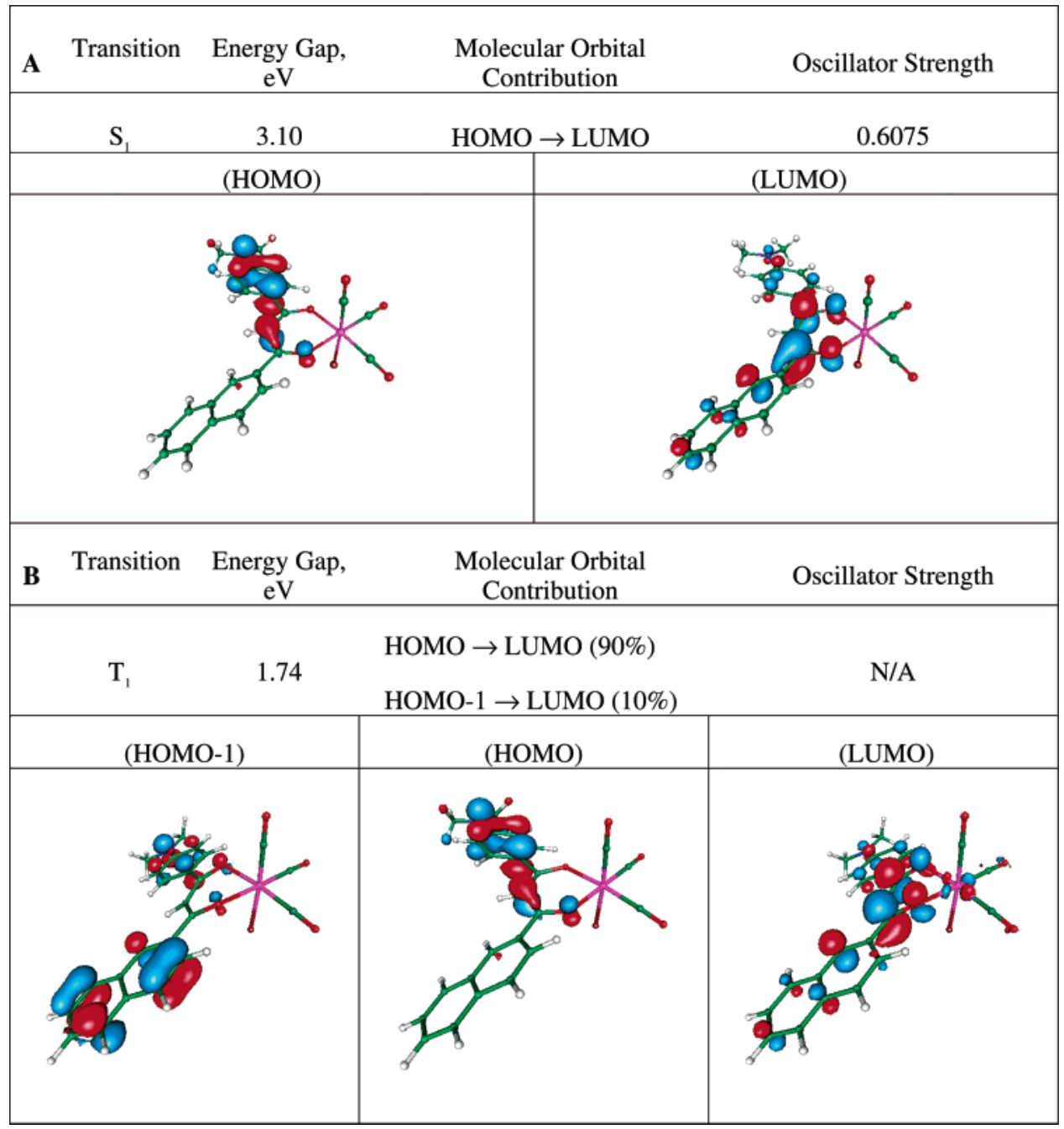

${ }^{a}$ A. TD-B3LYP method based on the geometry optimized $\mathrm{S}_{0}$ state (B3LYP). B. TD-B3LYP method based on the geometry optimized $\mathrm{T}_{1}$ state (UB3LYP)

As shown in Table 1, a similar ICT reaction was observed for $\mathbf{1 a}$, in which the axial ligand in $\mathbf{1}$ is replaced by $\mathrm{CF}_{3} \mathrm{CO}_{2}{ }^{-}$, and the dipolar changes for $\mathrm{S}_{1}$ and $\mathrm{T}_{1}$ were derived to be 17.8 and $11.8 \mathrm{D}$, respectively. In comparison, only fluorescence solvatochromism resulting from ICT in the excited singlet state was observed for boron complex 2 , and the calculated dipolar change of $18.4 \mathrm{D}$ is consistent with that in $\mathbf{1}$ (or 1a) (Supporting Information).

The appreciable difference in the dipolar change is intriguing, as it may imply the intrinsic difference in the electron density distribution between $S_{1}$ and $T_{1}$ for both 1 and 1a. To show such a possibility, Table 2 depicts the composition and structures of the crucial frontier molecular orbitals for $\mathbf{1}$ involved in the transition of low-lying excited states using the TD-B3LYP// B3LYP/6-31G(d', p') method (Supporting Information). With this method, the Franck-Condon excitation energy from the ground state to the $S_{1}$ state was calculated to be $3.1 \mathrm{eV}$ (400 $\mathrm{nm})$, which is qualitatively consistent with the corresponding experimental results of $424 \mathrm{~nm}\left(\mathrm{~S}_{1}\right)$ in cyclohexane. Since the phosphorescence originates from the geometry-relaxed $\mathrm{T}_{1}$ state, we also performed the geometry optimization of the $T_{1}$ state, followed by a single-point calculation of the $\mathrm{T}_{1}-\mathrm{S}_{0}$ transition. As shown in Table 2, differences in the frontier orbital configuration between $S_{1}$ and $T_{1}$ are immediately discernible. The HOMO and LUMO frontier orbitals in the singlet manifold possess a $\pi$ configuration in the amino phenyl ligand and a $\pi^{*}$ pattern mainly in the 2-naphthyl ligand. One thus would expect that the $S_{0} \rightarrow S_{1}$ transition incorporated an electron-transfer process from the amino phenyl group to the 2-naphthyl moiety. ${ }^{11}$ In contrast, although to a large extent $(90 \%)$ similar HOMO and LUMO are involved in the $\mathrm{S}_{0} \rightarrow \mathrm{T}_{1}$ transition, contribution from other frontier orbitals are also substantial. The involvement of HOMO-1 orbital possessing a $\pi$ configuration of the 2-naphthyl and MLCT is $\sim 10 \%$ (Supporting Information). Different extents of charge transfer character render distinctly different solvatochromism effects, as observed experimentally.

The success in probing TSICT in complexes $\mathbf{1}$ and $\mathbf{1 a}$ warrants the design of other TSICT prototypes, among which complex 3 bearing various degrees of donor/acceptor substituents (Scheme 1) should receive considerable attention due to their facile syntheses. ${ }^{12}$ The $\mathrm{Os}(\mathrm{CO})_{n}$ fragment can thus be exploited as a building block to probe other types of reactions in the triplet manifold. For example, theoretical approaches have predicted that, relative to the fast excited-state proton transfer (ESPT) in the singlet state, the triplet-state proton transfer should be much slower due to the decrease in proton acidity. ${ }^{13}$ Unfortunately, cases of triplet state proton transfer are very rare ${ }^{14}$ because of the fast, dominant proton-transfer process in the singlet-excited state. If the rate of $\mathrm{S}_{1} \rightarrow \mathrm{T}_{n}$ ISC can compete with the proton-transfer rate in a singlet manifold, studies of 
the triplet-state proton-transfer become feasible. Accordingly, a proposed structure like complex $\mathbf{4}$, in which the phenol-ligand is expected to undergo ESPT, forming a quinone isomer, ${ }^{15}$ should be intriguing to probe the proton-transfer dynamics in the triplet state manifold. We thus believe that results presented in this study can spark a broad range of interest in both fundamental approach and synthetic field relevant to third-row transition metal complexes.

Acknowledgment. This work was supported by the National Science Council, Taiwan, R.O.C. (NSC93-2119-M-002-002). We thank the National Center for High-Performance Computing, Taiwan, for the use of their facility.

Supporting Information Available: Syntheses, photophysical experimental details, the Lippert-Mataga plot, and crystal data of 2. This material is available free of charge via the Internet at http://pubs.acs.org.

\section{References and Notes}

(1) (a) Baldo, M. A.; O'Brien, D. F.; You, Y.; Shoustikov, A.; Sibley, S.; Thompson, M. E.; Forrest, S. R. Nature 1998, 395, 151. (b) Welter, S.; Brunner, K.; Hofstraat, J. W.; De Cola, L. Nature 2003, 421, 54.

(2) (a) Liu, Y.; Jiang, S.; Glusac, K.; Powell, D. H.; Anderson, D. F.; Schanze, K. S. J. Am. Chem. Soc. 2002, 124, 12412. (b) Haskins-Glusac, K.; Ghiviriga, I.; Abboud, K. A.; Schanze, K. S. J. Phys. Chem. B 2004 108, 4969. (c) McMillin, D. R.; Moore, J. J. Coord. Chem. Rev. 2002, 229, 113. (d) Amini, A.; Harriman, A.; Mayeux, A. Phys. Chem. Chem. Phys. 2004, 6, 1157.
(3) (a) Lamansky, S.; Djurovich, P.; Murphy, D.; Abdel-Razzaq, F.; Lee, H.-E.; Adachi, C.; Burrows, P. E.; Forrest, S. R.; Thompson, M. E. J. Am. Chem. Soc. 2001, 123, 4304. (b) Brooks, J.; Babayan, Y.; Lamansky, S.; Djurovich, P. I.; Tsyba, I.; Bau, R.; Thompson, M. E. Inorg. Chem. 2002, 41, 3055.

(4) (a) Tyson, D. S.; Luman, C. R.; Zhou, X.; Castellano, F. N. Inorg. Chem. 2001, 40, 4063. (b) Song, L.-Q.; Feng, J.; Wang, X.-S.; Yu, J.-H.; Hou, Y.-J.; Xie, P.-H.; Zhang, B.-W.; Xiang, J.-F.; Ai, X.-C.; Zhang, J.-P. Inorg. Chem. 2003, 42, 3393. (c) Goodall, W.; Williams, J. A. G. Chem. Commun. 2001, 2514. (e) Del Guerzo, A.; Leroy, S.; Fages, F.; Schmehl, R. H. Inorg. Chem. 2002, 41, 359.

(5) (a) Shtykov, S.; Melnikov, G.; Goryacheva, I. J. Mol. Struct. 1999, 482-483, 699. (b) Murillo Pulgarin, J. A.; Molina, A. A.; Alanon Pardo, M. T. Anal. Chim. Acta 2000, 423(1), 85. (c) Perez-Ruiz, T.; MartinezLozano, C.; Tomas, V.; Martin, J. J. Pharm. Biomed. Anal. 2003, 32(2), 225.

(6) Sato, Y.; Morimoto, M.; Segawa, H.; Shimidzu, T. J. Phys. Chem. $1995,99,35$ 4580 .

(7) Zalis, S.; Farrell, I. R.; Vlcek, A. J. Am. Chem. Soc. 2003, 125,

(8) Chen, Y.-L.; Sinha, C.; Chen, I.-C.; Liu, K.-L.; Chi, Y.; Yu, J.-K.; Chou, P.-T.; Lu, T.-H. Chem. Commun. 2003, 3046.

(9) $\Delta f$ is the solvent polarity parameter function and is generally expressed as $\Delta f=(\epsilon-1) /(2 \epsilon+1)$, where $\epsilon$ denotes the static dielectric constant of the solvent (Supporting Information).

(10) Lakowicz, J. R. Principles of Fluorescence Spectroscopy; Plenum Press: New York, 1983.

(11) Meyer, T. J. Pure Appl. Chem. 1986, 50, 1293.

(12) Yu, W.-S.; Cheng, C.-C.; Cheng, Y.-M.; Wu, P.-C.; Song, Y.-H.; Chi, Y.; Chou, P.-T. J. Am. Chem. Soc. 2003, 125, 10800.

(13) For example, see Scheiner, S. J. Phys. Chem. A 2000, 104, 5898.

(14) Grellmann, K. H.; Mordzinski, A.; Heinrich, A. Chem. Phys. 1989, 136, 201.

(15) 15. Kaczmarek, L.; Borowicz, P.; Grabowska, A.; J. Photochem. Photobiol. A: Chem. 2001, 138, 159, and references therein. 